\title{
Server-Side Caching Strategies for Online Auction Sites
}

\author{
Daniel A. Menascé ${ }^{1}$ and Vasudeva Akula ${ }^{2}$ \\ 1 Department of Computer Science, \\ George Mason University, Fairfax, VA 22030, USA \\ menasce@cs.gmu.edu \\ 2 School of Information Technology \& Engineering George Mason University, \\ Fairfax, VA 22030, USA \\ vakula@gmu.edu
}

\begin{abstract}
Online auction sites have very specific workloads and user behavior characteristics. Previous studies on workload characterization conducted by the authors showed that i) bidding activity on auctions increases considerably after $90 \%$ of an auction's life time has elapsed, ii) a very large percentage of auctions have a relatively low number of bids and bidders and a very small percentage of auctions have a high number of bids and bidders, iii) prices rise very fast after an auction has lasted more than $90 \%$ of its life time. Thus, if bidders are not able to successfully bid at the very last moments of an auction because of site overload, the final price may not be as high as it could be and sellers, and consequently the auction site, may lose revenue. In this paper, we propose server-side caching strategies in which cache placement and replacement policies are based on auction-related parameters such as number of bids placed or percent remaining time till closing time. A main-memory auction cache at the application server can be used to reduce accesses to the back-end database server. Trace-based simulations were used to evaluate these caching strategies in terms of cache hit ratio and cache efficiency.
\end{abstract}

\section{Introduction}

Online auctions are becoming an important segment of the e-commerce space with large players such as eBay and Yahoo!Auctions. It has been observed that web requests follow Zipf-like distributions and that this fact can be used to design caches that improve hit ratios [3]. That work was applied to web sites that mostly served static pages. E-commerce sites generate most of their pages dynamically. Our workload characterization work [1,10] of online auction sites also found evidences of Zipf distributions, and power laws in general. Our previous work also showed that the workload of online auction sites is substantially different from that of online retailers and uncovered a plethora of interesting findings that can be used, among other things, to improve the performance of online auction sites. These findings include i) A very large percentage of auctions have a relatively low number of bids and bidders and a very small percentage of 
auctions have a high number of bids and bidders. ii) There is some bidding activity at the beginning stages of an auction. This activity slows down in the middle and increases considerably after $90 \%$ of an auction's life time has elapsed. iii) Prices rise faster in the first $20 \%$ of an auction's life time than in the next $70 \%$ of its life time. However, after the age of an auction reaches $90 \%$, prices increase much faster than in the two previous phases. iv) A relatively few users are responsible for winning the majority of auctions. v) A relatively few sellers are responsible for creating the majority of the auctions. vi) The majority of bids are placed by a relatively small number of unique bidders.

We rely on these facts to suggest that a main memory auction cache at the application server can save a significant number of accesses to a backend database and thus significantly reduce the server-side latency for both read and write requests at online auctions sites. We propose several cache placement and replacement policies. We conducted an experimental validation of our policies for various cache sizes using a trace derived from a data collection process in which an agent collected data for over 340,000 auctions from Yahoo!auctions. We measured the cache hit ratio and the cache efficiency. Our findings show that small caches can be quite efficient and are able to provide reasonably large hit ratios.

Significant work has been done in the area of web caching [5, 6, 13, 14]. Many conferences including IEEE's International Workshop on Web Content Caching and Distribution (WCW), already in its $10^{\text {th }}$ installement, and the International World Wide Web Conference (WWW), started in 1994, have been some of the preferred venues for cache-related publications. A good collection of cache related information and resources is Brian Davidson's web site (www.web-caching.com/). In general, web caching work can be classified into browser caching, client-side proxy caching, network caching (as in Content Delivery Networks), and serverside caching. Our work falls into the realm of server-side caching and is specific to online auctions sites. The authors are not aware of any other auction-site specific caching study that uses real traces from a large production auction site.

The rest of this paper is organized as follows. Section two provides some background and definitions used throughout the paper and introduces in more detail the notion of an auction cache. Section three describes typical user behavior using a Customer Behavior Model Graph. The next section describes the cache placement and replacement policies studied here. Section five presents and discusses the results of our experiments. Finally, section six presents some concluding remarks.

\section{Background}

An open auction (i.e., one that is still in progress) is defined by several parameters including: opening time, $t_{o}$, closing time, $t_{c}$, and number of bids, $b(t)$, submitted up to time $t$. From these parameters, one can define $t_{p}(t)$, the percent remaining closing time at time $t$, as $\left[\left(t_{c}-t\right) /\left(t_{c}-t_{o}\right)\right] \times 100$.

The typical software architecture of an auction site is multitiered and is composed of three layers as indicated in Fig. 1. The first layer comprises web 


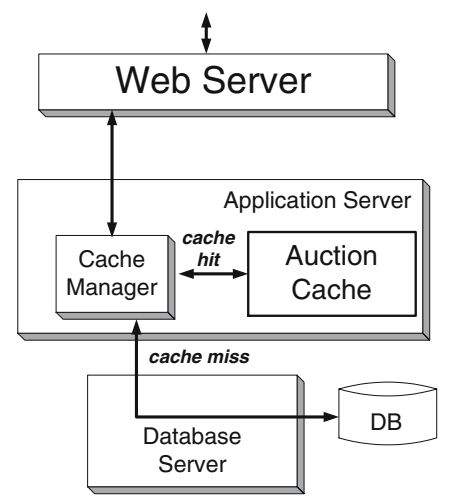

Fig. 1. Architecture of an online auction site

servers that handle the incoming HTTP requests and serve static HTML pages. Most pages served by an auction site are dynamically generated by an application server, which implements the site's business logic. The application server may need to access persistent data stored in a backend database server. An example is the processing of a request to view all bids for a given auction. The bid information comes from the database. The application server then generates an HTML page with the bid information. This page is passed back to the web server, which sends the page back to the browser.

The performance of online auction sites can potentially be improved if a fraction of the millions of auctions is cached in the main memory of the application server, thus avoiding most of the trips to the database. As indicated in Fig. 1, when the information about an auction can be found in the main memory cache of the application server, a cache hit occurs. Otherwise a cache miss occurs and the information about the auction has to be retrieved from the database. The auction cache can store the following information about an auction:

- Auction information: includes headline, textual description, names of the files that store thumbnail images and possibly larger images, number of bids, highest bid, highest bidder ID, bid increment, starting price, and seller ID.

- Bid history: all the bids placed for the auction. Each bid includes bidder ID, overall bid sequential number, bid price, and proxy flag (indicates if the bid was manually placed or if it was placed by an agent on behalf of the bidder).

- Seller information: specifies the seller of the auction and his/her rating information, number of auctions sold, and date since the seller has been a member of the auction site.

In this paper we are only concerned with transactions that can potentially be served by an auction cache. Therefore, we do not consider transactions such as auction creation, registration, and login. The transactions that may be served by the cache can be divided into read-only and write transactions. Read-only transactions include View Bid, View Auction Information, View Auction Details 
(e.g., description of items, warranty), View Seller Information and Ratings, and View Comments on the Seller. The main write transaction is Place Bid. There could be a problem executing write transactions at the cache without refreshing the database immediately. If a power failure occurs, the contents of the main memory cache could be lost and with it all updates (i.e., all bids on the cached auctions). However, large production e-commerce sites maintain multiple levels of backup power supplies. Typically, the servers have dual power supplies, from two separate power sources. There are multiple uninterrupted power supply (UPS) units that act as backup for these power supplies. In case of failure of these UPS units, or in cases in which the actual power supply is lost for a longer duration which can lead to the shutdown of UPS units, power generators act as backups for the UPS units. These generators can run for as long as the diesel supply lasts. So, power failures at popular e-commerce sites are extremely rare. Therefore, we assume that the auction cache survives power failures. This assumption assures that write transactions can be executed at the cache.

Failures due to software crashes are also possible. To cope with them, one may write a log of all write transactions at the application server in parallel with writing into the cache. A background process can be used to refresh the backend database at regular intervals from the log.

Most production auction sites have several web servers and several application servers. Therefore, each application server will have its own auction cache. To deal with problems of cache coherence we assume that any given auction can be cached at one and only one application server cache. This can be accomplished by identifying each auction by a unique id. Then, one can devise a function $f$ that takes as input an auction's unique id and returns the id of one of the application servers. The id of the application server that handles requests for a given auction can be first sent in a cookie and returned in a cookie to the web server so that the request can be dispatched to the proper application server.

Auction sites have grown rapidly in the last couple of years, and recent statistics indicate that eBay carries about 50 million items for sale at any time on its site [7. Yahoo!Japan carries 7.5 million items for sale [15]. We estimate that each auction, including its details, bids history and seller information, requires about $8 \mathrm{~KB}$ of cache space. Thus, to cache $5 \%$ of all auctions, an auction site carrying 10 million items for sale would require $10,000,000 \times 0.05 \times 8 \mathrm{~KB}=4 \mathrm{~GB}$ of main memory cache. This estimate does not include other items to cache, such as category names, state names, payment types and other static information to serve web requests quickly. Thus, for a caching scheme to be effective, it must provide a relatively high cache hit ratio for a reasonably small cache.

\section{User Behavior}

Users of an online auction site may invoke many different transactions during a session (i.e., a sequence of consecutive requests from a user during the same visit). We describe in what follows a typical user session using the Customer Behavior Model Graph (CBMG) defined by Menascé et al. 12. Each node of 


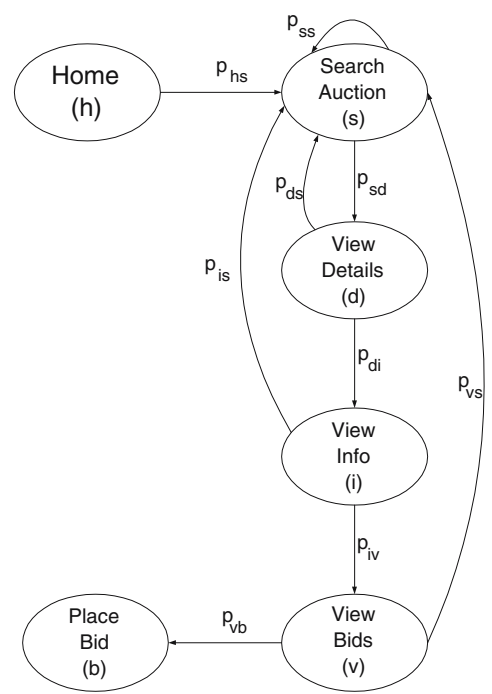

Fig. 2. Customer Behavior Model Graph (CBMG) for an online auction site

the graph represents a state in which a user may be during a session. Nodes of a graph are connected by directed arcs that indicate the possible transitions between states. Arcs are labeled with the probability that a transition between states occurs. Figure 2 depicts an example CBMG for an online auction site. Transactions that are not relevant to the cache are not shown. Transitions from each state to an Exit state are not shown for better readability. A CBMG can be used to determine the average number of visits per session to state $i, V_{i}$, as a function of the transition probabilities between states as shown in 12. Applying this method to the CBMG of Fig. 2, yields the following equations.

$$
\begin{gathered}
V_{s}=p_{h, s}+p_{s, s} V_{s}+p_{d, s} V_{d}+p_{i, s} V_{i}+p_{v, s} V_{v} \\
V_{d}=p_{s, d} V_{s} \\
V_{i}=p_{d, i} V_{d}=p_{d, i} p_{s, d} V_{s} \\
V_{v}=p_{i, v} V_{i}=p_{i, v} p_{d, i} p_{s, d} V_{s} \\
V_{b}=p_{v, b} V_{v}=p_{v, b} p_{i, v} p_{d, i} p_{s, d} V_{s}
\end{gathered}
$$

The value of $V_{s}$ can be obtained by solving Eqs. (1)-(5):

$$
V_{s}=\frac{1}{1-\left(p_{s, s}+p_{d, s} p_{s, d}+p_{i, s} p_{d, i} p_{s, d}+p_{v, s} p_{i, v} p_{d, i} p_{s, d}\right)}
$$

The ratio between read and write transactions, $R W$, is given by $R W=\left(V_{d}+\right.$ $\left.V_{i}+V_{v}\right) / V_{b}$. Using the values $p_{h, s}=1.0, p_{s, s}=0.8, p_{s, d}=0.1, p_{d, i}=0.55$, $p_{i, v}=0.5, p_{v, s}=0.75, p_{v, b}=0.2 p_{d, s}=0.4, p_{i, s}=0.45$, yields a value of RW equal to 33 . 


\section{Cache Placement and Replacement Policies}

A cache placement policy determines if an auction should be placed in the cache and a cache replacement policy determines which auction should be removed from the cache if the cache is full and a new auction needs to be cached.

The following cache placement policies have been evaluated.

- $\mathrm{AB} n$ : this is an activity based policy. An auction accessed at time $t$ is cached if $b(t) \geq n$ for that auction. For example the AB2 policy caches auctions with at least two bids already submitted at time $t$.

- PRTp: this is a percent remaining time policy. An auction is cached at time $t$ if $t_{p}(t)<p$.

- H-AND-n-p: this a hybrid policy that caches an auction if both $\mathrm{AB} n$ and PRT $p$ would cache it.

- H-OR-n-p: this is a hybrid policy that caches an auction if $\mathrm{AB} n$ or $\mathrm{PRT} p$ would cache it.

We consider the following auction replacement policies.

- AB: this replacement policy is only used in conjunction with the $\mathrm{AB} n$ placement policy. It removes the auction with the smallest number of submitted bids.

- PRT: this replacement policy is only used with the PRTn placement policy. It replaces from the cache the auction with the largest percent remaining time to close.

- AB-PRT: this replacement policy is used with both H-AND-n-p and H-OR$\mathrm{n}-\mathrm{p}$. It removes the auction with the largest percent remaining time to close among the ones with the smallest number of bids.

- Least Recently Used (LRU): replaces the auction that has received a request further in the past. This is a standard replacement policy used in operating systems [4].

- Least Frequently Used (LFU): replaces the auction that has received the smallest percentage of requests among all auctions in the cache. This policy has been considered in the context of operating systems [4] and in the web in combination with LRU 9].

A caching policy is then specified by its cache placement and replacement policy. We use the naming convention $<$ cache placement $>$ : $<$ cache replacement $>$ to name the caching policies investigated here. For example, AB2:LRU means that AB2 is the cache placement policy and LRU the replacement policy.

If the cache size is unlimited (i.e., at least as large as the total number of auctions), the replacement policy does not matter since all auctions will always find a place in the cache. Also, in this case, one should cache all auctions. This means that placement policies such as PRT100, which is a "always cache" policy, are optimal.

Figure 3 shows a relationship between the various policies in the unlimited cache size case. An arrow from policy $a$ to policy $b$ indicates that the hit ratio for $a$ is higher or the same as that of policy $b$ in the unlimited cache size case. 


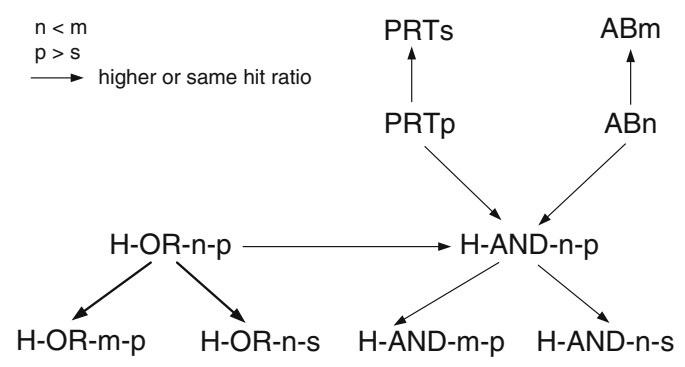

Fig. 3. Relation between placement policies for unlimited cache size

If the cache size is limited, there is a cost associated with evicting an auction from the cache since its state has to be refreshed in the backend database server. Also, an "always cache" policy may force a more popular auction than the incoming one out of the cache thus reducing the cache hit ratio.

\section{Experimental Evaluation}

The experimental evaluation of the policies described above was carried out with a trace-based simulation. The trace is derived from actual data collection performed for our previous workload characterization and closing time rescheduling work [1, 10, 11. The data collection process was described in detail in these papers. We provide here a brief summary of the process. A data collection agent gathered a total of 344,314 auction items created during the month of January 2003, belonging to over two thousand categories, from the Yahoo!Auctions site. A total of 1.12 million bids were placed on these auctions.

For this paper we used three weeks worth of that data, which contains 210,543 auctions, and 156,074 bids on these auctions. Note that the data we collected only contains the auction creation and bid records for each auction. Thus, we do not have data on read-only requests (e.g., view bids, view seller info, view auction details). However, for the purpose of the simulation, we inserted thirty read requests in the trace before each bid to maintain a RW ratio compatible with the CBMG analysis of section 3 ,

We varied the cache size to assess the impact of the placement and replacement policies as the cache size changed. We report the cache size as a percent, $P_{c}$, of the total number of auctions $N_{a}$. Thus, a $5 \%$ cache size implies that $5 \%$ of the 210,543 auctions can be stored in the cache. The amount of main memory required per auction is around 8,000 bytes.

Each experiment used a combination of cache placement and cache replacement policy and computed the following metrics for different cache sizes:

- Cache hit ratio $(H)$ : percent of cacheable transactions that were served from the auction cache.

- Cache efficiency $(\varepsilon)$ : defined as the ratio $(H \times 100) / N_{c}$, where $N_{c}$ is the average number of auctions in the cache. This number is computed, using 
Little's Law 8], as the product of the average cache throughput (i.e., number of auctions that leave the cache divided by the duration of the experiment) and the average time spent in the cache per auction. The cache efficiency $\varepsilon$ measures how much hit ratio one gets per cached auction on average.

Due to space limitations we only present a small subset of all graphs we generated in our simulations. A general observation seen from all graphs is that a relatively small cache, e.g., 4 to $5 \%$ of all auctions is enough to generate cache hit ratios of around 50 to $70 \%$. Even very small caches of about $1 \%$ can generate cache hit ratios as big as $40 \%$.

\subsection{Results for ABn Placement Policies}

Figure 4 displays the cache hit ratio for the $\mathrm{AB} 1: \mathrm{AB}, \mathrm{AB} 2 \mathrm{AB}$, and $\mathrm{AB} 3: \mathrm{AB}$ policies as a function of the percent cache size. The figure indicates that the three policies have almost the same cache hit ratio for very small cache sizes. However, as the cache size increases, $\mathrm{AB} 1 \mathrm{AB}$ outperforms $\mathrm{AB} 2 \mathrm{AB}$, which outperforms AB3:AB, as discussed above. It can also be observed that the cache hit ratio increases much faster at the beginning for smaller cache sizes. It can also be observed that a cache size of $0.5 \%$ is sufficient to provide a cache hit ratio of about $35 \%$ for all three policies. It can also be seen that all three policies approach very fast their unlimited cache size performance. For example, the $\mathrm{AB} 1 \mathrm{AB}$ policy has a limiting hit ratio of $68.2 \%$. Ninety nine percent of this value is achieved for a $4 \%$ cache size. Around $86 \%$ of the limiting performance of $\mathrm{AB} 1: \mathrm{AB}$ is obtained for a $2 \%$ cache size. While $\mathrm{AB} 1 \mathrm{AB}$ outperforms $\mathrm{AB} 2 \mathrm{AB}$, which outperforms $\mathrm{AB} 3 \mathrm{AB}$, the situation is reversed with respect to efficiency. For example, for a $P_{c}=0.25 \%$, the efficiency of $\mathrm{AB} 1 \mathrm{AB}$ is 0.078 while that of $\mathrm{AB} 3: \mathrm{AB}$ is 0.086 .

Figure 5 shows a comparison among the replacement policies AB, LRU, and LFU when used in conjunction with placement policy AB1, the best in Figure 4 .

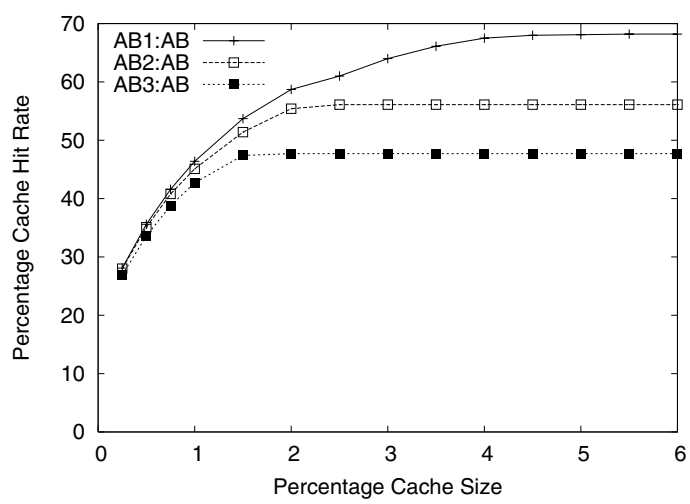

Fig. 4. Hit ratio for $\mathrm{AB} 1 \mathrm{AB}, \mathrm{AB} 2 \mathrm{AB}$, and $\mathrm{AB} 3: \mathrm{AB}$ 


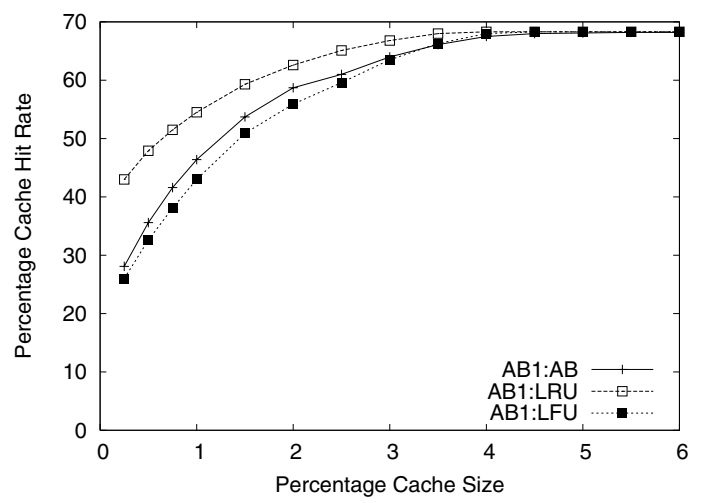

Fig. 5. Comparison among replacement policies AB, LRU, and LFU for placement policy AB1

As expected, for sufficiently large cache sizes, i.e., for $P_{c}>4.5 \%$ all three policies yield the same result. Smaller cache sizes distinguish the three policies with LRU being the best, followed by AB, and then by LFU. For example, for $P_{c}=$ $0.25 \%$, LRU outperforms $\mathrm{AB}$ as a replacement policy by a $53 \%$ margin. In fact, $\mathrm{AB} 1: \mathrm{LRU}$ has a $43 \%$ hit ratio while $\mathrm{AB} 1 \mathrm{AB}$ has a $28.1 \%$ hit ratio for $P_{c}=$ $0.25 \%$.

\subsection{Results for PRTp Placement Policies}

Figure 6 compares policies PRT10:PRT, PRT30:PRT, and PRT50:PRT. As was the case with the $\mathrm{AB} n$ placement policy, the increase in hit ratio is much faster for smaller caches. The unlimited cache size hit ratios for these policies are $18.6 \%$, $32.2 \%$, and $44.2 \%$, respectively, and occurs for a value of $P_{c}$ equal to $6 \%$. The

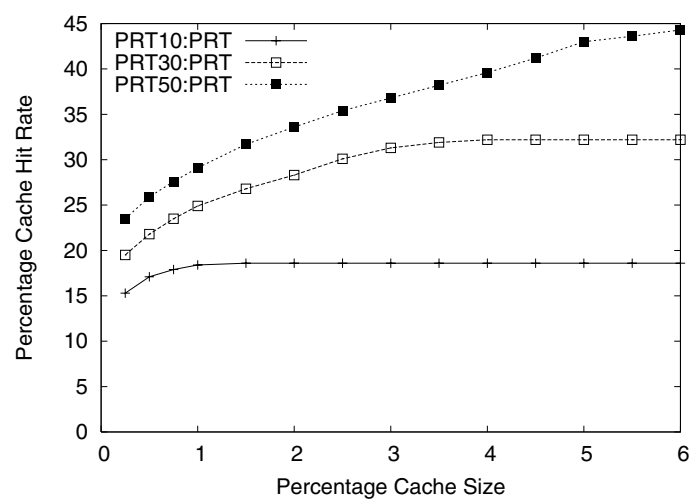

Fig. 6. Hit ratio for PRT10:PRT, PRT30:PRT, and PRT:50 


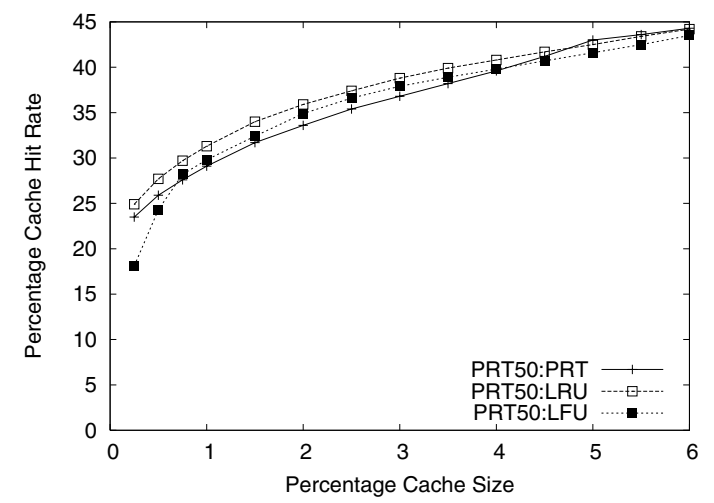

Fig. 7. Comparison among replacement policies PRT, LRU, and LFU for placement policy PRT50

difference in hit ratio is significantly reduced for smaller cache sizes. For example, for $P_{c}=0.25 \%$ the hit ratios for PRT10:PRT, PRT30:PRT, and PRT50:PRT, are $15.3 \%, 19.5 \%$, and $23.5 \%$, respectively. Figure 7 compares the effects of the PRT, LRU, and LFU replacement policies combined with the PRT50 placement policy. For a percent cache size of $6 \%$ all three replacement policies display similar results because the unlimited cache size behavior is already achieved at this point. For smaller cache sizes, LRU is slightly better than PRT, which is slightly better than LFU. The difference between these replacement policies when combined with PRT is not as marked as in the $\mathrm{AB} n$ case.

\subsection{Results for the Hybrid Policies}

We consider now policies of the type H-AND- $n-p$ :AB-PRT in Fig. 8, The unlimited cache size behavior is achieved for much smaller cache sizes than in the $\mathrm{AB} n$ and PRTp cases $\left(P_{c}=2.5 \%\right.$ instead of $\left.P_{c}=6.0 \%\right)$. As indicated in Fig. 3 , one would expect that for large cache sizes, the following orders, from best to worst, to hold: i) H-AND-1-30 $\rightarrow$ H-AND-2-30 $\rightarrow$ H-AND-3-30; ii) H-AND-1-50 $\rightarrow$ H-AND-2-50. iii) H-AND-1-50 $\rightarrow$ H-AND-1-30. iv) H-AND-2-50 $\rightarrow$ H-AND2-30. These relationships are confirmed in the graph of Fig. 8. However, Fig. 3 does not allow us to infer a relationship between H-AND-1-30 and H-AND-2-50. Our experiments indicate, as shown in Fig. 8, that H-AND-2-50 outperforms H-AND-1-30. For $P_{c}=2.5 \%$ the hit ratio of H-AND-2-50 is $15 \%$ higher than that of H-AND-1-30 and for a small cache of $P_{c}=0.3 \%$, the hit ratio of H-AND$2-50$ is $20.0 \%$ higher than that of H-AND-1-30. This means that it is better to start caching earlier as long as the auction seems to be more popular. Figure 9] is similar to Fig. 8 except that a H-OR placement policy is used as opposed to a $\mathrm{H}$-AND one. The H-OR policies provide higher hit ratios than their H-AND counterparts. We already knew that would be the case for unlimited cache sizes according to Fig. 3. For example, while H-AND-1-50:AB-PRT has a cache hit 


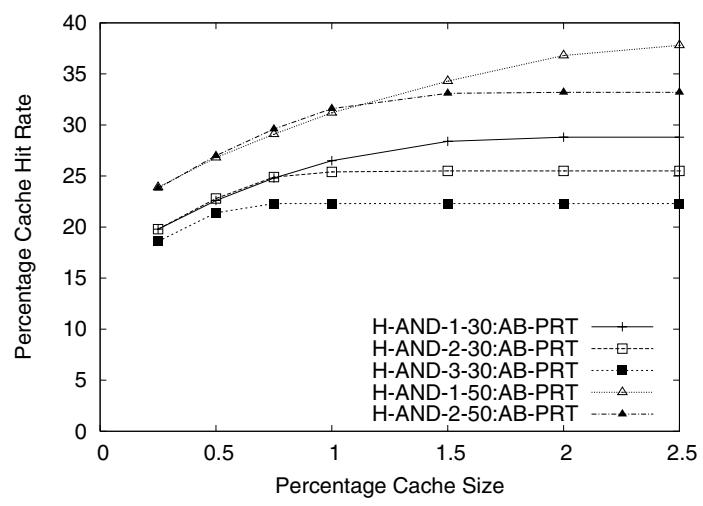

Fig. 8. Hit ratio for various HIT-AND- $n-p$ policies

ratio of $37.8 \%$ for $P_{c}=2.5 \%$, H-OR-1-50:AB-PRT has a cache hit ratio of $59.0 \%$ for the same value of $P_{c}$. Consider now the same relationship for a small cache size of $P_{c}=0.3 \%$. The H-OR-1-50 policy outperforms the H-AND-1-50 one by a factor of 1.7 .

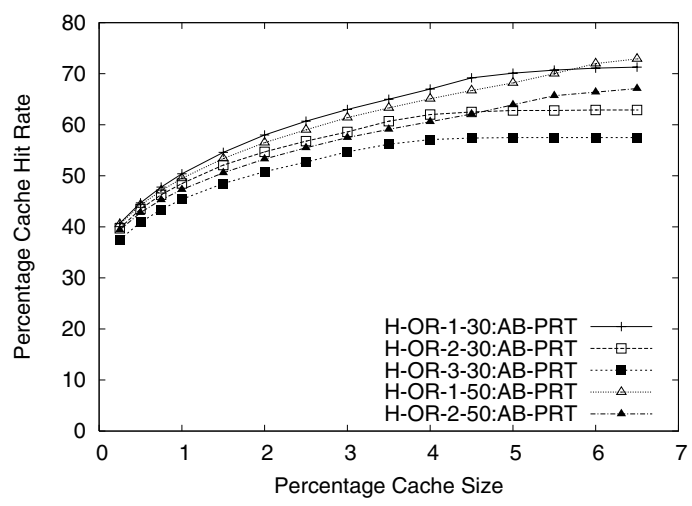

Fig. 9. Hit ratio for various HIT-OR- $n-p$ policies

Another observation from Fig. 9 is that a large cache is required to achieve an unlimited cache size performance level $\left(P_{c}=6.5 \%\right.$ as opposed to $\left.P_{c}=2.5 \%\right)$ when compared to the H-AND case. For the large cache sizes, the following relationships, which are in accordance with Fig. 3. can be observed (from best to worst): i) H-OR-1-50 $\rightarrow$ H-OR-2-50. ii) H-OR-1-30 $\rightarrow$ H-OR-2-30 $\rightarrow$ H-OR-330. iii) H-OR-1-50 $\rightarrow$ H-OR-1-30. iv) H-OR-2-50 $\rightarrow \mathrm{H}-\mathrm{OR}-2-30$. It is interesting to note the crossovers between H-OR-1-50 and H-OR-1-30 and between H-OR-250 and H-OR-2-30. They show that for smaller cache sizes, the preferred policy is not the one that has the best performance for unlimited cache sizes. 


\subsection{Policy Comparisons}

This section compares the best policies of each category - AB1:LRU, PRT50:LRU, H-OR-1-50:LRU, and H-AND-1-50:LRU — with respect to the cache hit ratio $H$ and the cache efficiency $\varepsilon$. Figure 10 compares these policies with respect to $H$ and shows that AB1:LRU and H-OR-1-50:LRU are very similar and far superior than PRT50:LRU and H-AND-1-50:LRU. AB1:LRU is slighty superior than H-OR-150:LRU for $0.25 \% \leq P_{c} \leq 4.0 \%$. For $P_{c}>4.0 \%$, H-OR-1-50:LRU has a higher hit ratio. Figure 11 compares the same policies in terms of cache efficiency. It can be seen that, for all four policies, $\varepsilon$ decreases very fast as the cache size increases indicating that small caches are very effective because of the power law characteristics of auction workloads [1, 10. In fact, the efficiency curves also follows a power law. For example, using regression on the AB1:LRU efficiency curve yields $\varepsilon=0.001704 / P_{c}^{0.703}$. As can be seen, AB1:LRU and H-OR-1-50:LRU have higher cache efficiency than the two other policies.

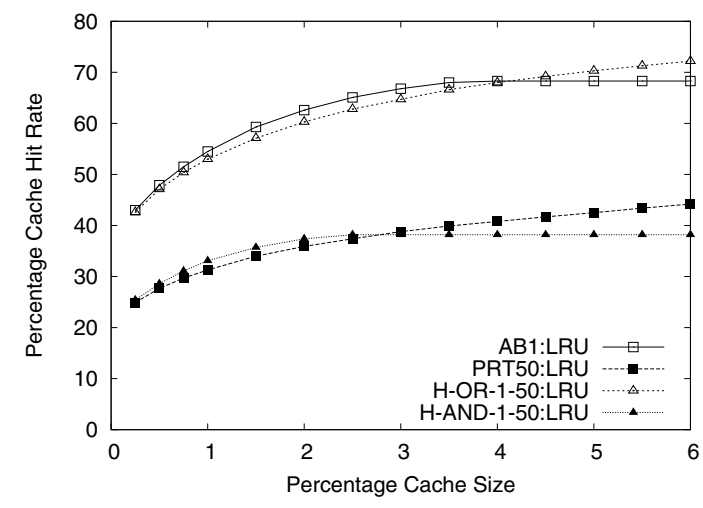

Fig. 10. Hit ratio comparison for various policies

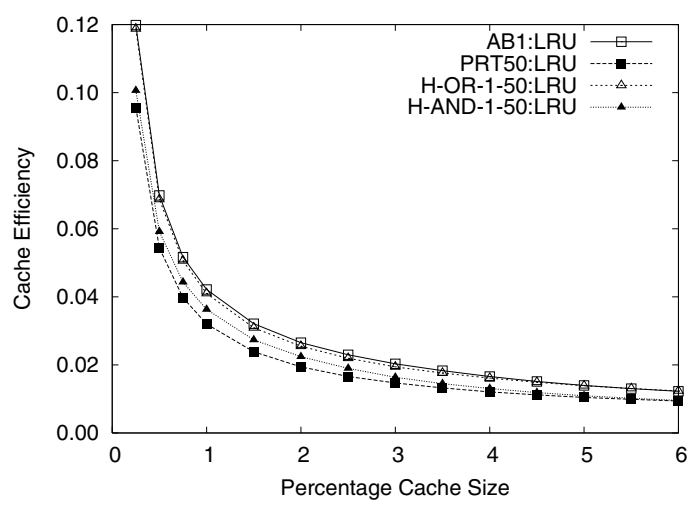

Fig. 11. Cache effectiveness comparison for various policies 


\section{Concluding Remarks}

This paper proposed several cache placement and replacement policies and analyzed them through trace-based simulations using traces from a large production site. Some general observations from our analysis are: i) LRU is the cache replacement policy that outperforms the others when combined with any of the placement policies considered. For example, for $P_{c}=0.25 \%$, AB1:LRU outperforms $\mathrm{AB} 1: \mathrm{AB}$ by a $53 \%$ margin. ii) Hit ratios increase much faster for small cache sizes than for larger ones. For example, for the AB1:AB policy, an increase in cache size from $0.25 \%$ to $0.5 \%$ provides a $27 \%$ increase in hit ratio. An increase in cache size from $4 \%$ to $4.5 \%$ provides only a $0.7 \%$ increase in hit ratio. iii) The unlimited cache behavior can be obtained with cache sizes ranging from $2.5 \%$, for H-AND policies, to around $6 \%$ for the other policies. iv) a relatively small cache, e.g., 4 to $5 \%$ of all auctions is enough to generate cache hit ratios of around 50 to $70 \%$ as is the case with the $\mathrm{AB} n$ policies. Even very small caches of about $1 \%$ can generate cache hit ratios as large as $40 \%$. v) the H-OR- $n-p$ policies provide a much better hit ratio than the H-AND- $n-p$ ones. For example, while H-AND-1-50:AB-PRT has a cache hit ratio of $37.8 \%$ for $P_{c}=2.5 \%$, H-OR-150:AB-PRT has a cache hit ratio of $59.0 \%$ for the same value of $P_{c}$. vi) The H-OR policies have crossover points indicating that the best policy for unlimited cache sizes is not the best for smaller cache sizes. vii) AB1:LRU and H-OR-1-50:LRU are very similar and far superior than PRT50:LRU and H-AND-1-50:LRU. viii) AB1:LRU is slighty superior than H-OR-1-50:LRU for $0.25 \% \leq P_{c} \leq 4.0 \%$. For $P_{c}>4.0 \%$, H-OR-1-50:LRU has a higher hit ratio. ix) The cache efficiency decreases very fast as the cache size increases indicating that small caches are very effective because of the power law characteristics of auction workloads. In fact, the efficiency curves also follows a power law.

In summary, the performance of online auction sites can be significantly increased with very small caches (on the order of $1 \%$ of the millions of items being auctioned). These small caches are more effective than larger caches and produce more hits per cached auction. We are currently designing a comprehensive benchmark for online auctions based on our workload characterization of such sites. We are also implementing a testbed compliant with this benchmark and we will be using it to test the caching policies described in this paper.

\section{References}

1. Akula, V., Menascé, D.A.: An analysis of bidding activity in online auctions. 5th Intl. Conf. (EC-Web 2004), Zaragoza, Spain, Aug./Sept. (2004) 206-217

2. Bapna,R., Goes, P., Gupta, A.: Online auctions: insights and analysis. Comm. ACM. 44(11) (2001) 42-50

3. Breslau, L., Cao, P., Fan, Li., Phillips, G., Shenker, S.:, Web Caching and Zipf-like Distributions: Evidence and Implications. INFOCOM (1), (1999), 126-134.

4. Coffman Jr., E.G., Denning, P.J.: Operating Systems Theory. Prentice Hall, Upper Saddle River, NJ (1973) 
5. Davison, B. D.: The Design and Evaluation of Web Prefetching and Caching Techniques PhD Dissertation, Rutgers University, October (2002) URL: http://citeseer.ist.psu.edu/davison02design.html

6. Douglis, F., Davison, B. D. (eds) : Web Content Caching and Distribution Proc. 8th International Workshop. Kluwer, June (2004)

7. hardwarezone.com: 10,000 Ebay Enthusiasts to Gather For 10TH Anniversary Celebration. URL: www.hardwarezone.com/news/view.php?id=1576\&cid=5, May 26 (2005)

8. Kleinrock, L.: Queuing Systems: Theory, Vol I. John Wiley \& Sons, NY (1975)

9. Lee, D., Choi, J., Kim, J., Noh, S., Min, S.L., Cho, Y., Kim, C.: LRFU: A Spectrum of Policies that Subsumes the Least Recently Used and Least Frequently Used Policies. IEEE Trans. Computers 50(12): 1352-1361 (2001)

10. Menascé, D.A., Akula, V.: Towards workload characterization of auction sites. Proc. IEEE 6th Annual Workshop on Workload Characterization (WWC-6), Austin, TX, Oct. 27 (2003)

11. Menascé, D.A., Akula, V.: Improving the performance of online auction sites through closing time rescheduling. 1st Intl. Conf Quantitative Evaluation of SysTems (QEST-2004), Enschede, the Netherlands, Sept. 27-30 (2004)

12. Menascé, D.A., Almeida, V.A., Fonseca, R., Mendes, M.: A methodology for workload characterization for e-commerce servers. ACM Conf. Electronic Commerce, Denver, CO, Nov. 3-5, (1999) 119-128

13. Pierre, G.: A Web caching bibliography, June (2000) URL : citeseer.ist.psu.edu/pierre00web.html

14. Yagoub, K., Florescu, D., Issarny, V., Valduriez, P.: Caching Strategies for Data-Intensive Web Sites. The VLDB Journal, (2000) 188-199. URL : citeseer.ist.psu.edu/yagoub00caching.html

15. Yahoo!Japan: Monthly Disclosure - March (2005 URL:http://ir.yahoo.co.jp/en/monthly/200503.html, April 8 (2005) 